\title{
Clinical Presentation, Course, and Prognostic Factors in Lymphocyte-Predominant Hodgkin's Disease and Lymphocyte-Rich Classical Hodgkin's Disease: Report From the European Task Force on Lymphoma Project on Lymphocyte-Predominant Hodgkin's Disease
}

By Volker Diehl, Michael Sextro, Jeremy Franklin, Martin-Leo Hansmann, Nancy Harris, Elaine Jaffe, Sibrand Poppema, Martin Harris, Kaarle Franssila, Jan van Krieken, Theresa Marafioti, loannis A nagnostopoulos, and Harald Stein

\begin{abstract}
Purpose: Recentstudies have suggested that lymphocyte-predominant Hodgkin's disease (LPHD) is both clinically and pathologically distinct from other forms of Hodgkin's disease, including classical Hodgkin's disease (CHD). How ever, large-scale clinical studies were lacking. This multicenter, retrospective study investigated the clinical characteristics and course of LPHD patients and lymphocyte-rich classical Hodgkin's disease (LRCHD) patients classified according to morphologic and immunophenotypic criteria.

Materials and Methods: Clinical data and biopsy material of all available cases initially submitted as LPHD w ere collected from 17 European and American centers, stained, and reclassified by ex pert pathologists.

Results: The 426 assessable cases were reclassified as LPHD (51\%), LRCHD (27\%), CHD (5\%), non-Hodgkin's lymphoma (3\%), and reactive lesion (3\%); $11 \%$ of cases were not assessable. Patients with LPHD and LRCHD were predominantly male, with early-stage disease and few risk factors. Patients with LRCHD were significantly older. Survival and failure-free survival rates with adequate therapy were similar for patients $w$ ith
\end{abstract}

$\mathbf{F}$ OR MORE THAN 50 years, efforts have been made to subclassify Hodgkin's disease (HD) pathologically. The underlying aims of these efforts were to establish reproducible as well as clinically meaningful categories and to understand better the underlying mechanisms of the disease. The most commonly adopted scheme is the Rye classification, which is a modification of the classification of

From the Department of Internal Medicine, University of Cologne, Germany; and the Pathology Departments of University of Frankfurt, Germany; Massachusetts General Hospital, Boston, MA; National Cancer Institute, Bethesda, MD; University of Groningen, the Netherlands; Christie Hospital, Manchester, United Kingdom; Helsinki University Central Hospital, Helsinki, Finland; Akademisch Ziekenhuis, Leiden, the Netherlands; and Klinikum Benjamin Franklin, Berlin, Germany.

Submitted July 14, 1998; accepted October 6, 1998.

Supported by a grant from the European Community.

Address reprint requests to Volker Diehl, MD, Klinik I für Innere Medizin, D-50924 Köln, Germany.

(1) 1999 by American Society of Clinical Oncology.

0732-183X/99/1703-0776
LPHD and LRCHD, and were stage-dependent and not significantly better than stage-comparable results for CHD (German trial data). Twenty-seven percent of relapsing LPHD patients had multiple relapses, which is significantly more than the $5 \%$ of relapsing LRCHD patients who had multiple relapses. Lymphocyte-predominant Hodgkin's disease patients had significantly superior survival after relapse compared w ith LRCHD or CHD patients; however, this was partly due to the younger average age of $\mathrm{PHD}$ patients.

Conclusion: The two subgroups of LPHD and LRCHD bore a close clinical resemblance that was distinct from CHD; the course was similar to that of comparable nodular sclerosis and mixed cellularity patients. Thorough staging is necessary to detect advanced disease in LPHD and LRCHD patients. The question of how to treat such patients, either by reducing treatment intensity or following a "watch and wait" approach, remains unanswered.

J Clin Oncol 17:776-783. ^ 1999 by American Society of Clinical Oncology.

Lukes and Butler ${ }^{1,2}$ established in 1966. Lymphocytepredominant Hodgkin's disease (LPHD) was defined as containing rare Reed-Sternberg-like cells mixed with atypical cells (referred to as "lymphocytic and histiocytic" type) in a background of great numbers of lymphocytes. In recent studies, LPHD accounts for 3\% to $8 \%$ of Hodgkin's cases in

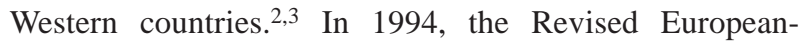
American Classification of Lymphoid Neoplasms (REAL classification) proposed the following categories of HD: nodular lymphocyte-predominant Hodgkin's disease, nodular sclerosis (NS), mixed cellularity (MC), lymphocyte depletion (LD), lymphocyte-rich classical HD (LRCHD; a provisional entity), and unclassifiable cases (UC). ${ }^{4}$ This classification system proposed that nodular lymphocytepredominant Hodgkin's disease is morphologically, biologically, and clinically distinct from other types of HD, termed classical HD (CHD), including NS, MC, LD, and LRCHD. Lymphocyte-predominant Hodgkin's disease was reported to present typically as early-stage disease, with slow progression and excellent outcome under standard therapy. A tendency toward more secondary non-Hodgkin's lympho- 
mas was noted, but this remained equivocal. ${ }^{5-7}$ The category of LRCHD was introduced as a provisional category for cases with a background consisting predominantly of lymphocytes, but with tumor cells of the classical Hodgkin's/ Reed-Sternberg type (CD30- and/or CD15-positive, but CD20-negative). In contrast, the tumor cells of LPHD express B-cell antigens such as CD20 and rarely express CD15 or CD30. It was postulated that LRCHD would behave similarly to CHD of the NS or MC type. The relative rarity of LRCHD and LPHD has so far prevented conclusive clinical studies. The multicenter effort reported here was designed to gain a better understanding from cases that were reviewed by expert pathologists in a homogeneous fashion.

In particular, the study aimed to answer the following questions regarding LPHD and LRCHD cases: (1) What is the initial presentation? (2) What is the clinical course, with respect to survival and failure-free survival, response, and relapse rates? (3) Do LPHD and LRCHD differ in clinical features? (4) Is there clinical evidence for a close relation to non-Hodgkin's lymphoma (NHL)? (5) What kind of clinical management can be recommended?

\section{MATERIALS AND METHODS}

\section{Selection of Cases}

Clinical data and biopsy material (paraffin blocks) of all available cases diagnosed initially as LPHD were collected from 17 European and American centers (Table 1), stained, and classified by a team of expert pathologists. Seven patients who were not treated or had surgery only and patients younger than 16 years were excluded from the analysis.

Table 1. Participating Centers

\begin{tabular}{lc}
\hline \multicolumn{1}{c}{ Center } & $\begin{array}{c}\text { N o. of } \\
\text { Assessable } \\
\text { Cases }\end{array}$ \\
\hline Christie Hospital, Manchester, United Kingdom & 90 \\
Helsinki University Central Hospital, Helsinki, Finland & 47 \\
German Hodgkin's Lymphoma Study G roup, Cologne, G ermany & 40 \\
Istituto N azionale per lo Studio e la Cura dei Tumori, Milan, Italy & 37 \\
Swedish N ational Health Care Programme, Uppsala, Sweden & 32 \\
Mayo Clinic, Rochester, M innesota, USA & 30 \\
Rigshospitalet, University of Copenhagen, Copenhagen, Denmark & 29 \\
Royal Victoria Infirmary, N ewcastle upon Tyne, United Kingdom & 26 \\
St. Bartholomew's Hospital, London, United Kingdom & 24 \\
A kademisch Ziekenhuis Leiden, Leiden, the N etherlands & 24 \\
M.D. A nderson Cancer Center, Houston, Texas, USA & 12 \\
Institut Bergonié, Bordeaux, France & 11 \\
Karolinska Hospital, Stockholm, Sweden & 11 \\
Hospital G regorio Marañón, Madrid, Spain & 6 \\
Università degli Studie “La Sapienza," Rome, Italy & 6 \\
Centre Hospitalier Lyon-Sud, Lyons, France & 1 \\
TO TA L & 426 \\
\hline
\end{tabular}

\section{Diagnostic Review Procedure}

The methods of the pathology panel review will be described in detail elsewhere. In brief, cases initially considered to be LPHD according to the Rye classification were newly classified according to morphologic and immunohistochemical criteria using the REAL classification. Eighty cases were excluded from further analysis for the following reasons: (1) the sample was too small, (2) immunostaining was technically inappropriate or impossible, or (3) no corresponding clinical data were available. The new diagnoses (REAL) of the 426 cases for which both clinical data and paraffin blocks were available were as follows: 51\% LPHD ( $n=219$ cases), 27\% LRCHD ( $n=115), 3 \%$ NHL $(\mathrm{n}=12), 5 \%$ CHD $(\mathrm{n}=19), 3 \%$ reactive lesions $(\mathrm{n}=14)$, and $11 \%$ technically inadequate sample $(n=47)$. All cases were reviewed without prior knowledge of any corresponding clinical data.

No significant differences in patient characteristics or survival results could be observed among the nodular, diffuse, or nodular-and-diffuse cases of LPHD. Therefore, these subgroups were pooled for this analysis into one single group of LPHD. Non-Hodgkin's lymphoma and CHD cases presented with a significantly higher stage and worse outcome. Details will not be presented here, because (1) these subgroups are too small for reliable analysis, and (2) they are not representative of CHD or NHL as a whole, because all cases had been initially diagnosed as LPHD (Rye). Some patients with reactive lesions nevertheless had HD in later biopsies (4 of 11 patients), which could either mean that biopsies were not representative or preceded the development of a true malignancy.

The following clinical variables were collected centrally in Cologne: trial identifier (where appropriate), patient identifier, sex, age at diagnosis, laparotomy, stage, systemic symptoms, extranodal involvement, mediastinal mass, bulky disease, splenic involvement, infradiaphragmatic involvement, sites of organ involvement, start, end, type and result of primary therapy, relapse with date, stage and histology, vital status, date and cause of death, and date and status of last observation. No laboratory data were collected. Inconsistencies and incomplete data sets were corrected with the help of the participating centers as necessary. The therapy regimens were grouped into "MOPP-like" (mechlorethamine, vincristine, procarbazine, prednisone), "ABVDlike" (doxorubicin, bleomycin, vinblastine, 5-[3,3-dimethyl-1-triazeno]imidazole-4-carboxamide), "MOPP/ABVD-like," and "other"; MOPP/ ABVD-like comprised alternating as well as hybrid regimens. Statistical analyses were performed with the SPSS Software (SPSS Inc, Chicago, IL), Version 6.1 for Microsoft Windows. Differences in the distribution of variables were assessed using Pearson's $\chi^{2}$ test. KaplanMeier estimates were calculated for overall survival (SV) and failurefree survival (FFS); comparisons of failure time data used the log-rank test. All reported $P$ values are two-sided. Survival and FFS are defined as the time from diagnosis to death or the time from diagnosis to an event, respectively. Hodgkin's-specific events for FFS included not achieving a complete remission (CR) after primary treatment, relapse, and death from Hodgkin's disease or NHL. Nonspecific events included death from any other cause. Hodgkin's-specific measures were used for most analyses, because we are interested in the biology of the disease and not in treatment-related or other deaths; however, SV after relapse was analyzed nonspecifically. Exploratory multivariate analyses for the evaluation of individual contributions of potential prognostic factors (age, sex, stage, and B symptoms, together with diagnosis, morphologic, and immunohistologic characteristics as recorded by the pathology panel) were performed using Cox-regression. Survival analyses were repeated with a stratification for center and for decade; stratification did not significantly alter the results obtained. 
RESULTS

\section{Patient Characteristics}

Patient characteristics for LPHD and LRCHD cases are listed in Table 2. Patients with LPHD and LRCHD had similar presentations: most patients were male ( $74 \% v 69 \%)$, had stage I or II disease ( $80 \% v 70 \%)$, and had few or no risk factors. However, LRCHD patients had a higher median age (35 $v 43$ years), had a mediastinal mass more often (although still rarely) (7\% v 15\%), or had stage III disease (14\%v $24 \%)$. No differences in the distribution of risk factors could be observed when stage was considered (data not shown). Approximately one half of the patients of any stage were staged by laparotomy. Liver, bone marrow, and lung were the most frequently involved sites of organ involvement. Median observation time was 6.8 years for patients with LPHD and 8.2 years for patients with LRCHD.

\section{Therapy}

Most patients were treated in the 1980s. Patients were treated according to the protocols that were in effect at the time of diagnosis in the participating institutions.

Table 3 lists details about the treatment modality by disease stage. Chemotherapy was MOPP-like in $49 \%$ of LPHD and LRCHD patients, ABVD-like in 4\%, MOPP/ ABVD-like in $36 \%$, and was other than the above categories in $11 \%$; these proportions were similar for patients receiving chemotherapy alone and for those receiving combined

Table 2. Characteristics of LPHD and LRCHD Patients

\begin{tabular}{|c|c|c|c|c|c|}
\hline & \multicolumn{2}{|c|}{ LPHD } & \multicolumn{2}{|c|}{ LRCHD } & \multirow[b]{2}{*}{$P$} \\
\hline & No. & $\%$ & No. & $\%$ & \\
\hline$n$ & 219 & & 115 & & \\
\hline \multicolumn{6}{|l|}{ Age, years } \\
\hline$>50$ & & 18 & & 32 & .0045 \\
\hline Median & \multicolumn{2}{|c|}{35} & \multicolumn{2}{|c|}{43} & \\
\hline Male sex & & 74 & & 69 & NS \\
\hline Stage & & & & & NS \\
\hline I & & 53 & & 46 & \\
\hline$\|$ & & 28 & & 24 & \\
\hline III & & 14 & & 24 & \\
\hline IV & & 6 & & 6 & \\
\hline Stage I/ II infradiaphragmatic & & 24 & & 15 & .15 \\
\hline B-symptoms & & 10 & & 11 & NS \\
\hline Mediastinal mass & & 7 & & 15 & .041 \\
\hline Bulky disease* & & 13 & & 11 & NS \\
\hline Splenic involvement & & 8 & & 15 & .066 \\
\hline O rgan involvement & & & & & NS \\
\hline Liver & 6 & 3 & 3 & 3 & \\
\hline Bone marrow & 2 & 1 & 1 & 1 & \\
\hline Lung & 2 & 1 & 4 & 4 & \\
\hline Skeleton & 1 & 1 & 0 & & \\
\hline 0 ther & 5 & 2 & 3 & 3 & \\
\hline
\end{tabular}

Abbreviation: NS, not significant.

*Definitions of bulky disease varied among contributors.
Table 3. Therapy by Stage for LPHD and LRCHD Patients Combined

\begin{tabular}{lccc}
\hline & $\begin{array}{c}\text { Radiotherapy } \\
(\%)\end{array}$ & $\begin{array}{c}\text { Chemotherapy } \\
(\%)\end{array}$ & $\begin{array}{c}\text { Combined } \\
\text { Modality } \\
\text { Therapy } \\
(\%)\end{array}$ \\
\hline Stage I $(n=168)$ & 88 & 1 & 12 \\
Stage II $(n=88)$ & 57 & 6 & 38 \\
Stage III $(n=59)$ & 19 & 41 & 41 \\
Stage IV $(n=19)$ & 5 & 63 & 32 \\
\hline
\end{tabular}

modality treatment. On the whole, therapy was considered to be adequate for stage. Ninety-nine percent of stage I and $95 \%$ of stage II patients received radiotherapy or combined modality treatment. Eighty-one percent of stage III and 95\% of stage IV patients received chemotherapy or chemotherapy plus radiotherapy. There were no significant differences in primary treatment between LPHD and LRCHD patients according to stage (data not shown). If chemotherapy alone was given, 94\% of patients received MOPP-, ABVD-, or MOPP/ABVD-like regimens.

\section{Results of Therapy}

Primary treatment results in both groups were virtually identical, with $96 \%$ of LPHD and LRCHD patients experiencing a CR (Table 4). There were no significant differences in treatment results between LPHD and LRCHD in analyses restricted to patients who received radiotherapy alone, chemotherapy alone, or combined modality therapy (data not shown).

Figure 1 shows the corresponding HD-specific SV and FFS. Although survival is slightly worse for LRCHD patients, no significant difference was observed between these groups $(P=.067$ for $\mathrm{SV} ; P=.57$ for FFS). Table 5 lists the 8-year Kaplan-Meier estimates for FFS and SV in LPHD and LRCHD, respectively, by stage. There were no statistically significant differences in SV or FFS between the two cohorts in an analysis stratified for stage. Early-stage patients in both groups had good-to-excellent survival, but treatment failures were common in both groups.

\begin{tabular}{|c|c|c|c|c|}
\hline & \multicolumn{2}{|c|}{ LPHD } & \multicolumn{2}{|c|}{ LRCHD } \\
\hline & No. & $\%$ & No. & $\%$ \\
\hline \multicolumn{5}{|l|}{ Result of primary therapy } \\
\hline Complete remission & 210 & 96 & 110 & 96 \\
\hline Partial remission & 6 & 3 & 0 & 0 \\
\hline Progressive disease/ no change & 3 & 1 & 2 & 2 \\
\hline Therapy not complete, unclear & 0 & 0 & 3 & 2 \\
\hline Relapse & 45 & 21 & 20 & 17 \\
\hline More than 1 relapse & 12 & $27 *$ & 1 & $5 *$ \\
\hline Death & 31 & 14 & 30 & 26 \\
\hline
\end{tabular}

*Percentage of relapsing patients $(P=.044)$. 

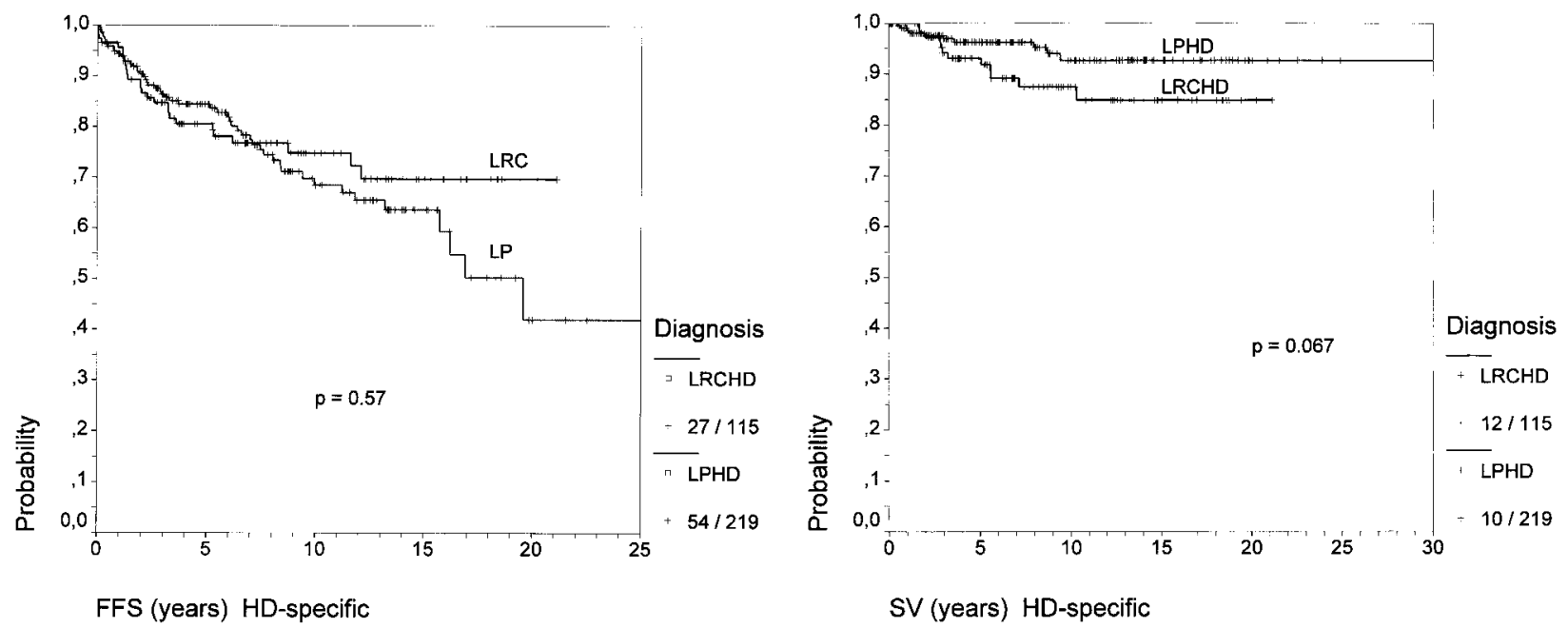

Fig 1. (left) Hodgkin's-specific failure-free survival for LPHD and LRCHD; (right) Hodgkin's-specific overall survival for LPHD and LRCHD.

\section{Relapses}

Twenty-one percent of LPHD patients compared with $17 \%$ of LRCHD patients experienced a first relapse after achieving CR (Table 4). Multiple relapses were observed in 12 of 45 relapsing patients (27\%) in the LPHD group, but in only one of 19 relapsed patients with initial LRCHD (5\%). Patients with LRCHD had a worse prognosis after relapse ( $P=.02$; Fig 2, left panel). Further analysis revealed that this difference could partly be explained by the older average age of LRCHD patients (Fig 2, right panel): the median ages (at first diagnosis) of relapsing LPHD and LRCHD patients were 34 and 40 years respectively; $9 \%$ and $25 \%$ of patients, respectively, were older than 55 years. Nevertheless, subgroup analysis of patients younger and older than 45 years in both groups revealed a favorable prognosis after relapse for younger patients with LPHD $(P=.020$; Fig 2B).

Table 5. Eight-Year HD-Specific Survival and Failure-Free Survival Kaplan-Meier Estimates With Standard Errors

\begin{tabular}{lcccc}
\hline \multicolumn{1}{c}{ Stage } & $\begin{array}{c}\text { LPHD } \\
(\%)\end{array}$ & $\begin{array}{c}\text { SE } \\
(\%)\end{array}$ & $\begin{array}{c}\text { LRCHD } \\
(\%)\end{array}$ & $\begin{array}{c}\text { SE } \\
(\%)\end{array}$ \\
\hline $\begin{array}{l}\text { Survival } \\
\text { I }\end{array}$ & 99 & 1.2 & 91 & 5.1 \\
II & 94 & 2.9 & 86 & 7.6 \\
III & 94 & 4.4 & 88 & 6.4 \\
IV & 41 & 30 & 67 & 19 \\
Freedom from treatment & & & & \\
$\quad$ failure & & & & \\
I & 85 & 4.3 & 81 & 6.1 \\
II & 71 & 7.3 & 76 & 8.7 \\
III & 62 & 9.8 & 74 & 8.5 \\
IV & 24 & 18 & 57 & 19 \\
\hline
\end{tabular}

\section{Causes of Death}

Thirty-one deaths (Table 6) were observed in the LPHD group, eight from HD (26\%) and 10 from secondary malignancies (32\%). In LRCHD patients, HD was the most common cause of death (33\%), and cardiovascular $(23 \%)$ and acute treatment-related $(13 \%)$ deaths occurred more frequently with LRCHD patients than with LPHD patients. Because of the small total number of deaths, the effect of treatment type on the cause-specific risk of death could not be analyzed. Data on fatal secondary malignancies are listed in Table 7.

In multivariate analysis, clinical prognostic factors for survival in LPHD and LRCHD were disease stage and age, with unfavorable prognosis associated with more advanced disease stage and older age at diagnosis. The absence of J-chain expression was the only other adverse prognostic factor that was confirmed by multivariate analysis of either SV or FFS, for all cases and for LPHD. The limited number of patients and events in each group prevented subgroup analyses.

\section{DISCUSSIO N}

In our review of 426 cases diagnosed initially as LPHD (Rye), $51 \%$ of cases were reclassified as LPHD and $27 \%$ were reclassified as CHD with a background of lymphocytes, termed LRCHD. The 219 patients with LPHD were predominantly male with early-stage disease and few adverse prognostic factors, thus confirming the observations of several previous studies.6-14 The 115 LRCHD patients presented similarly (male predominance, early-stage disease) but were older on average, and large mediastinal mass 


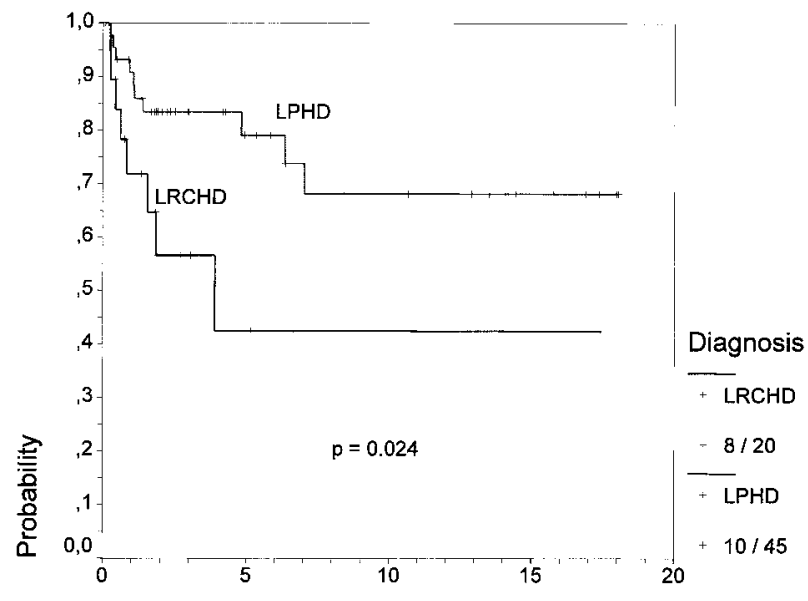

Survival after Relapse (years) non-specific

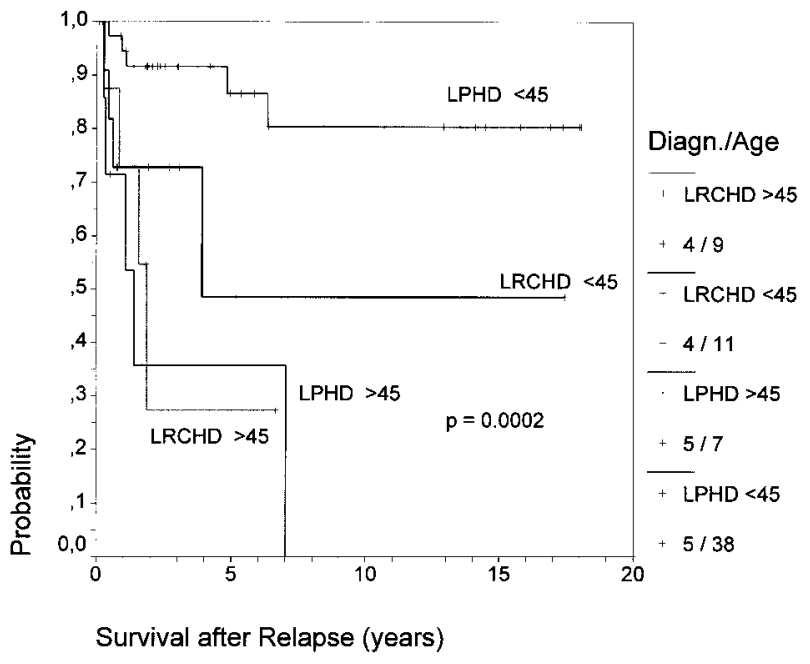

Survival after Relapse (years)

Fig 2. (left) Survival after relapse (nonspecific) for LPHD and LRCHD; (right) survival after relapse (nonspecific) for LPHD and LRCHD related to age (younger or older than 45 years).

was more frequent. Patients with LRCHD did not resemble patients with CHD in distribution of disease stage and prognostic factors.

Both groups have a good-to-excellent prognosis. Relapses were frequent in both groups, and patients continued to relapse within the observation period. Although Regula ${ }^{9}$ observed frequent late relapses in patients with LPHD, other authors ${ }^{7,10,11}$ have not uniformly confirmed this finding. The observed high frequency of relapse is not uncommon in early-stage CHD patients treated with radiotherapy. ${ }^{10,15,16}$ In our cohort, multiple relapses were more common and survival after relapse was slightly better in LPHD patients than in LRCHD patients, which may in part reflect a more benign character of relapse. However, LRCHD patients

\begin{tabular}{|c|c|c|c|c|}
\hline & \multicolumn{2}{|c|}{ LPHD } & \multicolumn{2}{|c|}{ LRCHD } \\
\hline & No. & $\%$ & No. & $\%$ \\
\hline HD & 8 & 3.7 & 10 & 8.7 \\
\hline \multicolumn{5}{|l|}{ Therapy } \\
\hline Primary & 0 & & 3 & 2.6 \\
\hline Salvage & 1 & 0.5 & 1 & 0.9 \\
\hline Cardiovascular & 4 & 1.8 & 7 & 6.1 \\
\hline \multicolumn{5}{|l|}{ Secondary tumors } \\
\hline A cute leukemia & 5 & 2.3 & 1 & 0.9 \\
\hline NHL & 2 & 0.9 & 2 & 1.7 \\
\hline Solid tumor & 3 & 1.4 & 1 & 0.9 \\
\hline \multicolumn{5}{|l|}{0 ther } \\
\hline Known & 6 & 2.7 & 2 & 1.7 \\
\hline Unknown & 1 & 0.5 & 1 & 0.9 \\
\hline Unknown, in CR & 1 & 0.5 & 2 & 1.7 \\
\hline Total deaths & 31 & 14 & 30 & 26 \\
\hline Total patients & 219 & 100 & 115 & 100 \\
\hline
\end{tabular}

were older than LPHD patients, and this may have substantially influenced prognosis. ${ }^{17}$

In the LPHD and LRCHD groups, there were almost as many deaths caused by secondary tumors as were caused by HD (14 v 18). These tumors caused $32 \%$ of deaths in the LPHD cohort, and only a minority of these deaths were caused by NHL. In cured HD, long-term toxicities (such as secondary malignancies, cardiovascular accidents, and infections) play an important role in survival. This risk is further increased with additional salvage therapy. ${ }^{18}$ In several reports, ${ }^{5,9,18}$ but not all, $, 10,11,13$ a higher probability of developing an NHL after LPHD has been described. Four of five patients experiencing secondary NHL reported by

Table 7. Data on Fatal Secondary Malignancies

\begin{tabular}{|c|c|c|c|c|c|}
\hline $\begin{array}{l}\text { Case } \\
\text { No. }\end{array}$ & Diagnosis & $\begin{array}{l}\text { Cause of } \\
\text { Death }\end{array}$ & $\begin{array}{l}\text { Age at } \\
\text { Death, } \\
\text { Years }\end{array}$ & $\begin{array}{l}\text { Primary } \\
\text { Therapy }\end{array}$ & Relapse \\
\hline 1 & LPHD & Leukemia/M DS & 77 & CMT & No \\
\hline 2 & LPHD & Leukemia/M DS & 62 & RT & Yes \\
\hline 3 & LPHD & Solid tumor & 67 & RT & No \\
\hline 4 & LPHD & Leukemia/M DS & 66 & RT & No \\
\hline 5 & LPHD & Leukemia/MDS & 34 & $\mathrm{CT}$ & Yes \\
\hline 6 & LPHD & Leukemia/M DS & 75 & RT & No \\
\hline 7 & LPHD & Solid tumor & 79 & $\mathrm{CT}$ & Yes \\
\hline 8 & LPHD & NHL & 57 & $\mathrm{CT}$ & No \\
\hline 9 & LPHD & NHL & 51 & RT & No \\
\hline 10 & LPHD & Solid tumor & 83 & RT & No \\
\hline 11 & LRCHD & Leukemia/M DS & 36 & $\mathrm{CMT}$ & No \\
\hline 12 & LRCHD & NHL & 72 & $\mathrm{CT}$ & No \\
\hline 13 & LRCHD & NHL & 45 & RT & Yes \\
\hline 14 & LRCHD & Solid tumor & 82 & RT & No \\
\hline
\end{tabular}


Miettinen did not receive irradiation or any kind of chemotherapy, which suggests that most of these NHLs were not treatment-related. Recently, a clonal relationship between large-cell lymphoma arising from LPHD and the initial tumor could be established. ${ }^{19,20}$ In other series, high rates of secondary NHL (together with the B-cell origin of the tumor cells) have led to speculation that LPHD is not HD, but a low-grade B-cell NHL. In our present series, only two patients with LPHD and two patients with LRCHD died from NHL. Additionally, four nonfatal occurrences of secondary NHL were documented, two directly after primary LPHD and two after one or more relapses of LPHD; there may have been other undocumented cases. This observed rate of $2.9 \%$ suggests that the probability of developing secondary NHL is increased in LPHD patients when compared with CHD patients. An analysis from the International Database on Hodgkin's Disease estimated a secondary NHL rate of $1.0 \%$ for all $\mathrm{HD}$ patients at 10 years after first diagnosis. ${ }^{18}$

Unlike low-grade NHL (eg, follicular lymphoma), most LPHD patients presented with early-stage disease and remained in CR. This behavior more strongly resembles that of classical HD than that of low-grade NHL of B-cell type. The high rate of cure, low rate of subsequent NHL or relapse, predominance of limited nodal disease, and relatively young age associated with LPHD do not fit into the standard pattern of, for example, follicle-center lymphomas. Recently, Kanzler and Bräuninger and others ${ }^{21-24}$ convincingly demonstrated that CHD as well as LPHD are both malignant B-cell lymphomas of germinal center origin. Thus, B-cell NHLs might well be expected to occur after $\mathrm{CHD}$, and some such cases were seen in our series. It would be interesting to investigate by single-cell analyses the clonal relationships of the original CHD or LPHD and the secondary NHL.

The fact that absence of J-chain seems to define a subgroup of LPHD cases with a poorer prognosis was unexpected. The test for J-chain was positive for most LPHD cases but negative in most cases of CHD. No attempt will be made in the present clinical report to interpret these observations conclusively. J-chain, a polypeptide that links immunoglobulin molecules into groups of two ( $\operatorname{IgA}$ ) or five ( $\operatorname{IgM})$, has been investigated in LPHD and CHD by various authors, ${ }^{25,26}$ but without relation to clinical characteristics. Kelenyi, ${ }^{27}$ however, reported that J-chain had significant prognostic power in multiple myeloma; as in the present analysis, positive cases had better results. Briefly, the presence of $\mathrm{J}$-chain indicates the ability of the cell to produce immunoglobulins and therefore shows that the cell is well differentiated and retains its functionality as a B cell.
This property would logically be expected to correlate with a lower degree of malignancy.

Several authors have reported a more benign course for paragranuloma or lymphocyte-predominant cases, and Miettinen reported an 80\% 10-year survival for untreated nodular LPHD cases. ${ }^{1,5,28-30}$ Since the publication of these reports, it has been speculated that therapy for LPHD could be reduced without increased hazard to the patient. In our series, all analyzed patients received standard treatment according to stage, thus we were unable to assess the additional benefit of standard treatment for LPHD cases compared with a "watchand-wait" strategy. However, for the clinical management of LPHD, it should be noted that stage III and IV disease was diagnosed in $20 \%$ of LPHD cases and $31 \%$ of LRCHD cases in our series. This implies that thorough staging is needed independently of the subtype of HD, as survival and freedom from treatment failure were substantially worse for advancedstage patients than for those with early-stage disease. The prognosis for both LPHD and LRCHD in this extensively reviewed cohort was no better than for stage-matched CHD (NS and MC cases from the German Hodgkin's Lymphoma Study Group, data not shown). From our data alone, there is no rationale for a less intensive treatment of LPHD. However, only a minority of patients in either group died from HD. This suggests that current treatment strategies might not be optimal in terms of late toxicity: the cumulative risk for a secondary tumor as well as cardiac or pulmonary death increases with time and might reverse the benefit of treatment in the long term. Unfortunately, there are still no prospective trials to test whether a reduction of therapy is safe for patients with LPHD. For any such study, multicenter efforts will be needed: the prevalence of LPHD and LRCHD is less than $5 \%$, and the 334 cases in this study come from a cohort of more than 6,000 cases of HD.

Patients with LPHD and LRCHD did not show the typical distribution of disease stage and risk factors found in patients with NS or MC. It remains unclear why the richness in lymphocytes correlates with slowly progressive earlystage disease. Lymphocyte-predominant Hodgkin's disease and LRCHD could not be distinguished morphologically, but only by sophisticated histopathology with the use of immunophenotyping. Relapses in patients with LRCHD occurred less frequently and were more often fatal than were relapses in patients with LPHD. Multiple relapses were relatively more frequent after LPHD than after LRCHD. These subtle differences were found in subgroup analyses and should thus be interpreted with caution.

This is the first report to present the clinical characteristics and prognosis of a large series of centrally reviewed LRCHD cases using the definition of the REAL classification system. Clinically, LRCHD had a closer resemblance to 
LPHD than to NS or MC. As we only assessed cases that came from a cohort of cases that were originally diagnosed as LPHD (Rye), the whole picture of LRCHD might be different after review of all subtypes of HD that might harbor cases now regarded as LRCHD. To clarify this issue, clinicopathologic studies of lymphocyte-rich cases from the MC and NS groups are needed. The review of project cases emphasized that the diagnosis of lymphoma should be confirmed by expert hematopathologists, thereby allowing adequate treatment for CHD, LPHD, or NHL.

Almost all of our patients had received, immediately after diagnosis, a therapy regimen that was appropriate to their initial presentation. A watch-and-wait strategy, in which no immediate therapy is given, has been tested for stage I follicular lymphoma patients who are without residual disease after surgery ${ }^{31}$ : the overall and relapse-free survival of irradiated and untreated patients were similar. The main advantage of a watch-and-wait approach would be the avoidance of side effects and late effects of radiotherapy or chemotherapy. Analyses have shown that although the HD-related death rate in patients treated for HD decreases during the years after diagnosis, the overall death rate remains above that of the general population, largely because of cardiac failures and secondary cancers. ${ }^{32}$ Concerning secondary cancers, however, one must distinguish between those induced by treatment (leukemia, solid tumor) and the NHLs, which are often a transformation of the initial LPHD. ${ }^{19,20,33}$ The latter would not be avoided by a watch-andwait policy and might even increase, because treatment might suppress the development of a transformed lymphoma. $^{34,35}$ It must also be remembered that, whereas follicular lymphoma patients have a median age of approximately 60 years and little prospect of long-term cure, LPHD patients are typically young, and excellent long-term survival rates are possible. To answer the question of whether patients with LPHD, at least in stage I, would fare well without immediate treatment, we propose a global study to compare a watch-and-wait strategy with current standard protocols.

\section{ACKN O W LEDG MENT}

We thank the following persons for their essential participation: (A) Contributing clinicians: A. Anselmo, U. Axdorph, M. Björkholm, G. Bonadonna, V. Bonfante, B. Coiffier, D. Crowther, H. Eghbali, A.M. Gianni, B. Glimelius, A. Gustavsson, T. Habermann, F.B. Hagemeister, T.A. Lister, F. Mandelli, E. Noordijk, S. Proctor, J. Radford, L. Specht, P. Taylor, and L. Teerenhovi. (B) Contributing pathologists: B. Angus, C. Baroni, F. Berger, M. Dictor, A. Georgii, M. Harris, K. Hou-Jensen, P. Kluin, P. Kurtin, A. MacDonald, J.A. McBride, J. Menarguez, A. Norton, A. Öst, S. Pilotti, W. Pugh, I. Soubeyran, and C. Sundström.

\section{REFEREN CES}

1. Lukes RJ, Butler JJ: The pathology and nomenclature of Hodgkin's disease. Cancer Res 26:1063-1081, 1966

2. Lukes RJ, Butler JJ, Hicks EB: Natural history of Hodgkin's disease as related to its pathologic picture. Cancer 19:317-344, 1966

3. Colby TV, Hoppe RT, Warnke RA: Hodgkin's disease: A clinicopathologic study of 659 cases. Cancer 49:1848-1858, 1981

4. Harris NL, Jaffe ES, Stein H, et al: A revised European-American classification of lymphoid neoplasms: A proposal from the International Lymphoma Study Group. Blood 84:1361-1392, 1994

5. Miettinen M, Franssila KO, Saxen E: Hodgkin's disease, lymphocyte predominance nodular: Increased risk of subsequent nonHodgkin's lymphoma. Cancer 51:2293-2300, 1983

6. Trudel MA, Krikorian JG, Neiman RS: Lymphocyte predominant Hodgkin's disease: A clinicopathologic reassessment. Cancer 59:99106, 1987

7. Pappa I, Norton AJ, Gupta RK, et al: Nodular type of lymphocyte predominant Hodgkin's disease. Ann Oncol 6:559-565, 1995

8. Hansmann ML, Zwingers T, Boeske A, et al: Clinical features of nodular paragranuloma (Hodgkin's disease, lymphocyte predominance type, nodular). J Cancer Res Clin Oncol 108:321-330, 1984

9. Regula DP, Hoppe RT, Weiss LM: Nodular and diffuse types of lymphocyte predominance Hodgkin's disease. N Engl J Med 318:214219, 1988

10. Borg-Grech A, Radford JA, Crowther D, et al: A comparative study of the nodular and diffuse variants of lymphocyte-predominant Hodgkin's disease. J Clin Oncol 7:1303-1309, 1989

11. Crennan E, D'Costa I, Liew KH, et al: Lymphocyte predominant Hodgkin's disease: A clinicopathologic comparative study of histologic and immunophenotypic subtypes. Int J Radiat Oncol Biol Phys 31:333-337, 1995

12. von Wasielewski R, Werner M, Fischer R, et al: Lymphocyte predominant Hodgkin's disease: An immunohistochemical analysis of 208 reviewed Hodgkin's disease cases from the German Hodgkin Study Group. Am J Pathol 150:793-803, 1997

13. Bodis S, Kraus MD, Pinkus G, et al: Clinical presentation and outcome in lymphocyte-predominant Hodgkin's disease. J Clin Oncol 15:3060-3066, 1997

14. Orlandi E, Lazzarino M, Brusamolino E, et al: Nodular lymphocyte predominance Hodgkin's disease: Long-term observation reveals a continuous pattern of recurrence. Leuk Lymphoma 26:359-368, 1997

15. Longo DL, Glatstein E, Duffey PL, et al: Radiation therapy versus combination chemotherapy in the treatment of early-stage Hodgkin's disease: Seven-year results of a prospective randomized trial. J Clin Oncol 9:906-917, 1991

16. Brusamolino E, Lazzarino M, Orlandi E, et al: Early-stage Hodgkin's disease: Long-term results with radiotherapy alone or combined radiotherapy and chemotherapy. Ann Oncol 5:101-106, 1994 (suppl 2)

17. Nordentoft AM, Pedersen-Bjergaard J, Brincker $H$, et al: Hodgkin's disease in Denmark: A national clinical study by the Danish Hodgkin study group, LYGRA. Scand J Haematol 24:321-334, 1980

18. Henry-Amar M: Second cancer after treatment of Hodgkin's disease: A report from the International Database on Hodgkin's Disease. Ann Oncol 3:117-128, 1992 (suppl 4)

19. Wickert RS, Weisenburger DD, Tierens, et al: Clonal relationship between lymphocytic predominance Hodgkin's disease and concurrent 
or subsequent large-cell lymphoma of B-lineage. Blood 86:2312-2320, 1995

20. Greiner TC: Nodular lymphocyte-predominant Hodgkin's disease associated with large-cell lymphoma: Analysis of Ig gene rearrangements by V-J polymerase chain reaction. Blood 88:657-666, 1996

21. Kanzler H, Hansmann ML, Kapp U, et al: Molecular single cell analysis demonstrates the derivation of a peripheral blood-derived cell line (L1236) from the Hodgkin/Reed-Sternberg cells of a Hodgkin's lymphoma patient. Blood 87:3429-3436, 1996

22. Bräuninger A, Küppers R, Strickler JG, et al: Hodgkin and Reed-Sternberg cells in lymphocyte predominance Hodgkin's disease represent clonal populations of germinal center-derived tumor cells. Proc Natl Acad Sci U S A 94:9337-9342, 1997

23. Marafioti T, Hummel M, Anagnostopoulos I, et al: Origin of nodular lymphocyte-predominant Hodgkin's disease from a clonal expansion of highly mutated germinal-center B cells. N Engl J Med 337:453-458, 1997

24. Ohno T, Stribley JA, Wu G, et al: Clonality in nodular lymphocyte-predominant Hodgkin's disease. N Engl J Med 337:459465, 1997

25. Poppema S: The diversity of the immunohistological staining pattern of Sternberg-Reed cells. J Histochem Cytochem 28:788-791, 1980

26. Stein H, Hansmann ML, Lennert K, et al: Reed-Sternberg and Hodgkin cells in lymphocyte-predominant Hodgkin's disease of nodular subtype contain J chain. Am J Clin Pathol 86:292-297, 1986
27. Kelenyi G: Intracellular J chains in lymphoproliferative diseases. Virchows Arch A Pathol Anat Histopathol 405:365-378, 1985

28. Gough J: Hodgkin's disease: A correlation of histopathology with survival. Int J Cancer 5:273-281, 1970

29. Smetana HF, Cohen BM: Mortality in relation to histiologic subtype in Hodgkin's disease. Blood 11:211-224, 1956

30. Keller AR, Kaplan HS, Lukes RJ, et al: Correlation of histopathology with other prognostic indicators in Hodgkin's disease. Cancer 22:487-499, 1968

31. Soubeyran P, Eghbali H, Trojani M, et al: Is there any place for a wait-and-see policy in stage I0 follicular lymphoma? A study of 43 consecutive patients in a single center. Ann Oncol 7:713-718, 1996

32. Cosset JM, Henry-Amar M, Meerwaldt JH: Long-term toxicity of early stages of Hodgkin's disease therapy: The EORTC experience. Ann Oncol 2:77-82, 1991 (suppl 2)

33. Hansmann M-L, Stein H, Fellbaum C, et al: Nodular paragranuloma can transform into high-grade malignant lymphoma of B type. Hum Pathol 20:1169-1175, 1989

34. Horning SJ, Rosenberg SA: The natural history of initially untreated low-grade non-Hodgkin's lymphomas. N Engl J Med 311: 1471-1475, 1984

35. Bastion Y, Sebban C, Berger F, et al: Incidence, predictive factors, and outcome of lymphoma transformation in follicular lymphoma patients. J Clin Oncol 15:1587-1594, 1997 